\title{
Power management in adjacent cognitive femtocells with distance-dependent interference in full coverage area
}

\author{
Guobin Zhang ${ }^{*}$, Xin Ao, Ping Yang and Ming Li
}

\begin{abstract}
In cognitive heterogeneous networks, when multiple adjacent femtocells are deployed, uncontrolled transmission power can lead to severe mutual interference. In order to reduce interference while satisfying capacity requirements, we propose a power management scheme for an adjacent femtocell network. Firstly, we build an interference model for adjacent femtocells, in which distance-dependent interference to terminals within the whole femtocell coverage area is considered. Then, we calculate the total capacity of adjacent femtocells based on the mutual interference and formulate a non-convex optimization problem to maximize the capacity under the transmission power constraints. To solve the non-convex problem, we divide it into two convex subproblems and solve them with the Lagrange dual theory and linear programming method. Finally, we derive the closed-form expression of the optimal power configuration to maximize capacity while minimizing energy consumption simultaneously. The simulation results indicate that the proposed power scheme demonstrates obvious improvement in terms of capacity and power economization, compared with the maximal power configuration method.
\end{abstract}

Keywords: Adjacent femtocells, Power management, Distance-dependent interference, Capacity optimization

\section{Introduction}

Recently, with the development of radio access technologies and mobile internet applications, the demand for mobile data services increases explosively [1]. Latest surveys show that more than $80 \%$ of mobile traffic is originated at home or work [2]. Therefore, traditional cellular networks are confronted with the challenge of enhancing the coverage and improving the capacity of indoor and hotspot areas. In allusion to this challenge, femtocell networks which offer better quality of experience (QoE) for indoor and hotspot users [3] are being considered as a promising technique to enhance the performance of cellular networks.

When multitudes of femtocells coexist, severe interference may arise between adjacent femtocells if the transmission power is uncontrolled. Currently, a lot of research efforts are focused on the power control and interference elimination issues in heterogeneous femtocell networks.

*Correspondence: guobinzh@163.com

Department of Electronic Engineering, Dongguan University of Technology, Daxue Road, Dongguan, China
Most of these researches establish a heterogeneous network structure model, analyze interference between different cells, and optimize power configuration under interference and maximal transmission constraints. The authors in [4-6] construct a heterogeneous network with one macro cell and multiple small cells. The small cells utilize the licensed spectrum of the macro cell through cognitive radio with the overlay style. The authors in $[7,8]$ build an interference model for a hierarchical heterogeneous network and optimize the time frequency resource allocation. The proposed interference model considers the interference of the licensed network to the second- and third-level networks, as well as the interference between the second- and third-level networks. The authors in $[8,9]$ aim to maximize the capacity of the cognitive network, considering the interference constraint of the licensed network and transmission power constraint of the cognitive network. The authors in [3] propose a resource allocation scheme for cognitive femtocells considering sub-channel occupation fairness, co-tier and cross-tier interference mitigation, and spectrum-sensing errors.

\section{至 Springer}

(c) 2016 Zhang et al. Open Access This article is distributed under the terms of the Creative Commons Attribution 4.0 International License (http://creativecommons.org/licenses/by/4.0/), which permits unrestricted use, distribution, and reproduction in any medium, provided you give appropriate credit to the original author(s) and the source, provide a link to the Creative Commons license, and indicate if changes were made. 
The main problems of the aforementioned researches are as follows: (1) Most literatures use the same approach in the analysis of the interference between femtocells, which seldom consider the distribution range and relative locations of femtocells. Therefore, few of them manage to analyze the interference and power control policy for adjacent femtocells, where severe interferences arise more frequently. (2) Few of the existing literatures discuss about the effect of user distribution and density upon network capacity, which is worthy of being considered in the process of power control for enhancing the network capacity. (3) The present interference model of a heterogeneous network is complex mainly due to the reason that all the femtocells are treated together rather than separately. As a result, the power optimization problem demands high computational complexity, and it is also hard to achieve a closed-form solution. What is worse, some researches [7-10] assume fixed channel gains from transmitters to receivers in spite of their positions. Obviously, this is against the common sense that channel gain varies with transmission distance. Therefore, building a distance-dependent interference model is a more rational option.

To reduce the interference between adjacent femtocells and enhance their sum capacity simultaneously, we propose a specialized power management scheme in this paper. In the scheme, we consider the distance-dependent variation of channel gain in the analysis of network interference and sum capacity. To maximize the sum capacity, a non-convex power optimization problem is formulated and a two-step solution is provided accordingly. The solution divides the original non-convex problem into two convex problems and solves them by the Lagrange dual theory and the linear programming theory. The main contributions of this paper are summarized as follows:

1. We propose an interference model considering a distance-dependent channel gain for adjacent femtocells.

2. When computing the sum capacity of femtocells, we take into account the user density for each femtocell and the signal to noise ratio (SNR) of users distributed in the whole coverage area of the femtocells.

3) We derive a closed-form expression of an optimal power configuration scheme for adjacent femtocells, which can maximize their sum capacity while satisfying respective capacity requirement and minimizing the total energy consumption.

The rest of this paper is organized as follows. In Section 2, we construct the system model including a two-level heterogeneous network and multiple adjacent femtocells. In Section 3, we build the distance-dependent interference model and formulate the problem of network capacity optimization. We also provide a two-step method for solving the problem and summarize the detailed algorithm for optimal power configuration. Simulation results for performance analysis and validation are given in Section 4, followed by the conclusion in Section 5.

\section{System model}

Similar to $[1,6,11-13]$, we consider a two-level heterogeneous cognitive network as shown in Fig. 1. The macro cell is at the first level, which occupies the licensed frequency. Multiple femtocells compose the second-level network, which adopt cognitive radio to sense the licensed frequency and find idle frequency to utilize.

The femtocell is deployed to cover the hot spots of a licensed network so as to enhance the communication quality of service (QoS) of a hot spot area [14, 15]. In a dense femtocell-deployed network, severe interference may exist between adjacent femtocells. Therefore, we construct the network model with two adjacent femtocells to analyze their mutual interference. As shown in Fig. 2, two femtocells named Femtocell 1 and Femtocell 2 cover two circles with the radius of $R_{1}$ and $R_{2}$, respectively. Their base stations are both located in the center of the coverage area. The distance between the base stations of Femtocell 1 and Femtocell 2 is denoted by $D$. According to [16], the interference gain varies with the distance from the user position to the femtocell base station. Without loss of generality, we randomly choose one point to analyze its received interference. The details will be provided later in Section 3. In Fig. 2, we assume $\operatorname{dot} A_{1}$ is the randomly chosen point, and we define $L_{1}$ to denote the distance from $A_{1}$ to the center point of Femtocell 1 and define $L_{2}$ to denote the distance from $A_{1}$ to the base station of Femtocell 2 . The angle between $L_{1}$ and $D$ is denoted by $\theta$. In Section 3, we will discuss the power configuration strategy between the two adjacent femtocells based on the model as shown in Fig. 2.

\section{Power configuration strategy}

In this section, we carry out a thorough analysis of the interference and capacity of adjacent femtocells and propose a detailed power management strategy to maximize the sum capacity while minimizing the power consumption.

\subsection{Interference and capacity analysis}

For wireless communication, the channel gain varies with the distance between transmitter and receiver. The larger the distance, the smaller the channel gain becomes. Hence, in femtocell networks, the channel gain changes with the distance between the current user and the base station of the adjacent femtocell. Assume that the transmission power of Femtocell 1 and Femtocell 2, as shown in Fig. 2 , is denoted by $P_{1}$ and $P_{2}$, respectively. Let $P_{1, A_{1}}$ 


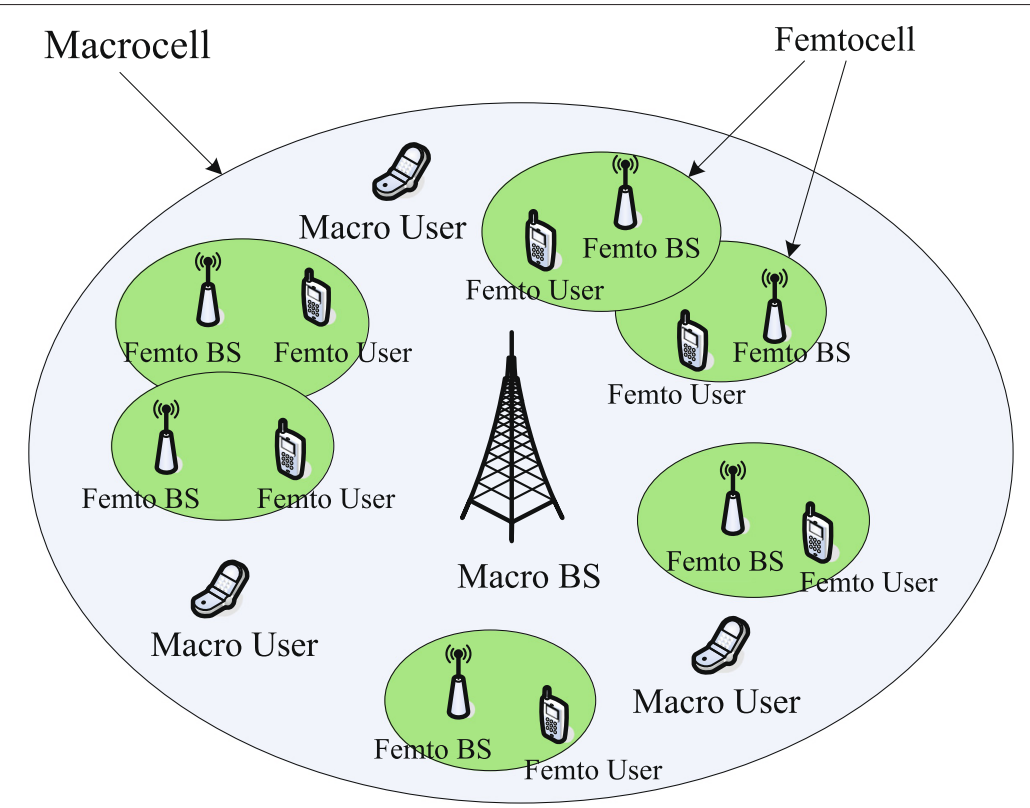

Fig. 1 Two-level heterogeneous cognitive network model. BS base station

and $P_{2, A_{1}}$ be the received power from the base station of Femtocell 1 and Femtocell 2 in $\operatorname{dot} A_{1}$, respectively. According to the literature [16], $P_{1, A_{1}}$ and $P_{2, A_{1}}$ can be expressed as the following, i.e.,

$$
\begin{aligned}
& P_{1, A_{1}}=P_{1} H_{1} C_{1} L_{1}^{-\alpha_{1}}, \\
& P_{2, A_{1}}=P_{2} H_{2} C_{2} L_{2}^{-\alpha_{2}} .
\end{aligned}
$$

In Eqs. (1) and (2), $H_{1}$ and $H_{2}$ are random variables capturing the small-scale fading value of the radio link from the base station of Femtocell 1 and Femtocell 2, respectively [16]. $C_{1}$ and $C_{2}$ indicate the path loss constant of
Femtocell 1 and Femtocell 2, respectively. $\alpha_{1}$ and $\alpha_{2}$ indicate the distance-related path loss exponent of Femtocell 1 and Femtocell 2, respectively.

Assume that the channel Gauss white noise power is $\sigma^{2}$ and the total interference received in $\operatorname{dot} A_{1}$ is $I_{1, A_{1}}$. Since the total interference includes received power from the base station of Femtocell 2 and Gauss white noise power, $I_{1, A_{1}}$ can be expressed as

$$
I_{1, A_{1}}=P_{2} H_{2} C_{2} L_{2}^{-\alpha_{2}}+\sigma^{2} .
$$

The SNR at $\operatorname{dot} A_{1}$ is defined as the ratio of the power received from the base station of Femtocell 1 to the total interference including received power from Femtocell 2

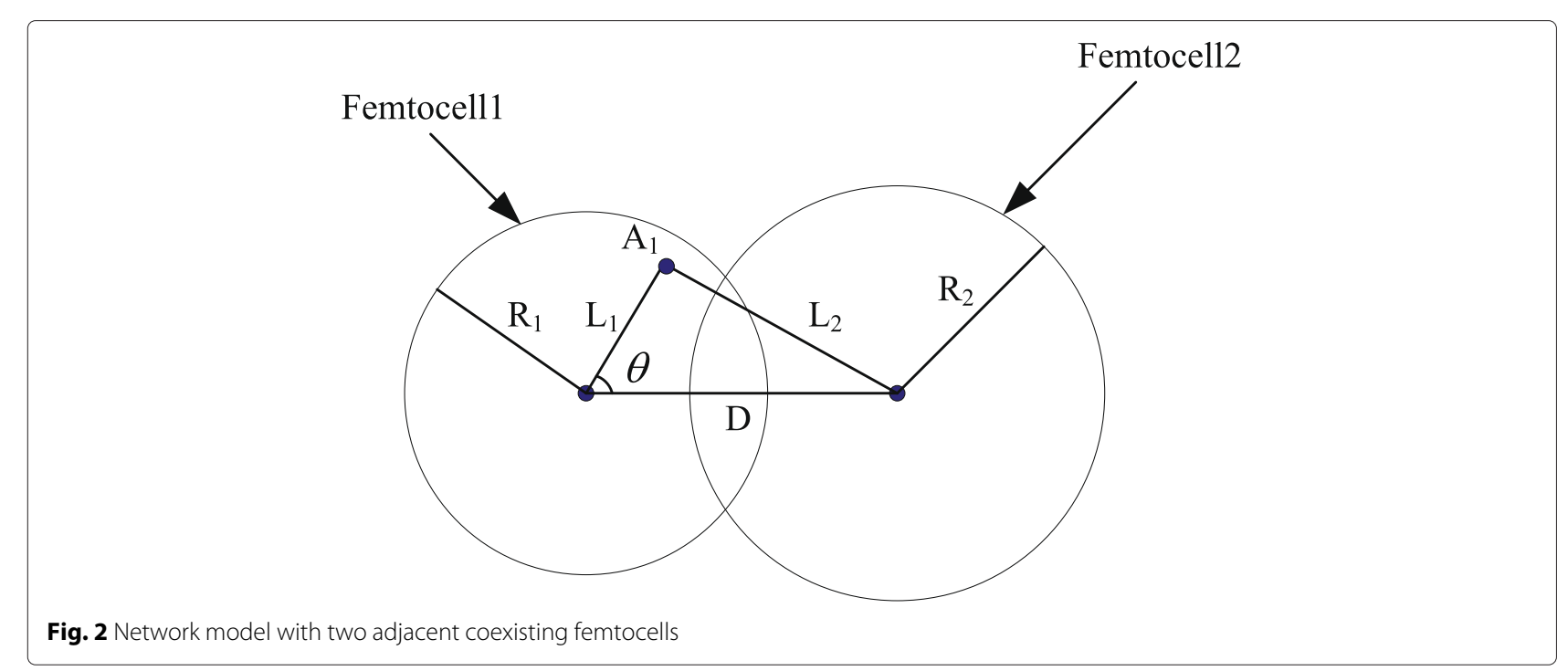


and Gauss white noise power. Therefore, expression (4) can be used to compute $\mathrm{SNR}_{1}$ which denotes the SNR at $\operatorname{dot} A_{1}$,

$$
\mathrm{SNR}_{1}=\frac{P_{1, A_{1}}}{I_{1, A_{1}}}=\frac{P_{1} H_{1} C_{1} L_{1}^{-\alpha_{1}}}{P_{2} H_{2} C_{2} L_{2}^{-\alpha_{2}}+\sigma^{2}} .
$$

The network capacity is defined as the summation of the communication rate of the terminals distributing within the coverage area. For simplicity, we compute the capacity of Femtocell 1 by the integral of the product of the user density and SNR in random position over the whole covering area of Femtocell 1. We define $\mathrm{Cap}_{1}$ indicating the capacity of Femtocell 1, which is expressed by

$$
\mathrm{Cap}_{1}=\int_{0}^{R_{1}} \int_{0}^{2 \pi} \frac{\rho_{1} P_{1} H_{1} C_{1} L_{1}^{-\alpha_{1}}}{P_{2} H_{2} C_{2} L_{2}^{-\alpha_{2}}+\sigma^{2}} L_{1} d \theta d L_{1}
$$

where $\rho_{1}$ denotes the user distribution density of Femtocell 1.

From Fig. 2, Eq. (6) can be obtained:

$$
L_{2}=\sqrt{\left(D-L_{1} \cos \theta\right)^{2}+\left(L_{1} \sin \theta\right)^{2}} .
$$

Next, Eq. (7) can be deduced from Eqs. (5) and (6).

Similarly, we define $\mathrm{Cap}_{2}$ to indicate the capacity of Femtocell 2 and $\rho_{2}$ to denote the user distribution density of Femtocell 2. And the same deduction method can be applied for the expression of $\mathrm{Cap}_{2}$, as shown in Eq. (8).

$$
\begin{aligned}
& \mathrm{Cap}_{1}= \\
& \int_{0}^{R_{1}} \int_{0}^{2 \pi} \frac{\rho_{1} P_{1} H_{1} C_{1} L_{1}^{-\alpha_{1}}}{P_{2} H_{2} C_{2}\left(\left(D-L_{1} \cos \theta\right)^{2}+\left(L_{1} \sin \theta\right)^{2}\right)^{-\frac{\alpha_{2}}{2}}+\sigma^{2}} L_{1} d \theta d L_{1}
\end{aligned}
$$

$$
\begin{aligned}
& \mathrm{Cap}_{2}= \\
& \int_{0}^{R_{2}} \int_{0}^{2 \pi} \frac{\rho_{2} P_{2} H_{2} C_{2} L_{2}^{-\alpha_{2}}}{P_{1} H_{1} C_{1}\left(\left(D-L_{2} \cos \theta\right)^{2}+\left(L_{2} \sin \theta\right)^{2}\right)^{-\frac{\alpha_{1}}{2}}+\sigma^{2}} L_{2} d \theta d L_{2}
\end{aligned}
$$

Finally, the sum capacity of Femtocell 1 and Femtocell 2 can be expressed by

$$
\mathrm{C}_{\text {tot }}=\mathrm{Cap}_{1}+\mathrm{Cap}_{2} \text {, }
$$

where $\mathrm{C}_{\text {tot }}$ refers to the sum capacity.

\subsection{Network capacity optimization}

In a cell centered with the base station, the transmission power is always expected to be as large as possible so as to obtain high capacity. However, in femtocell networks, large transmission power can bring severe interference to adjacent femtocells and reduce their capacity consequently. Therefore, we need to obtain the trade-off between the femtocell capacity and the interference to its adjacent femtocell, so as to maximize the sum net- work capacity. According to Eq. (9), femtocell capacity as follows:

$$
\max _{P_{1}, P_{2}}\left(\mathrm{Cap}_{1}+\mathrm{Cap}_{2}\right)
$$

subject to

$$
\begin{aligned}
& \mathrm{Cap}_{1} \geq \mathrm{Th}_{1} \\
& \mathrm{Cap}_{2} \geq \mathrm{Th}_{2} \\
& P_{1} \leq P_{\mathrm{LT} 1} \\
& P_{2} \leq P_{\mathrm{LT} 2}
\end{aligned}
$$

$$
P_{1} h_{1 p}+P_{2} h_{2 p} \leq I_{p} .
$$

In Eqs. (11) and (12), $\mathrm{Th}_{1}$ and $\mathrm{Th}_{2}$ denote the minimal capacity requirements of Femtocell 1 and Femtocell 2, respectively. In Eqs. (13) and (14), $P_{\mathrm{LT} 1}$ and $P_{\mathrm{LT} 2}$ denote the maximal permitted transmission power of Femtocell 1 and Femtocell 2, respectively. In Eq. (15), $h_{1 p}$ and $h_{2 p}$ denote the interference gain to licensed network from Femtocell 1 and Femtocell 2, respectively, and $I_{p}$ denotes the maximal interference power constraint to licensed network.

\subsection{Solution to optimization problem}

The formulated optimization problem contains a complex integral expression in the objective and constraint conditions. Therefore, it is difficult to solve it with the normal method for convex optimization problems. Herein, we adopt a two-step method to solve it. First, we transform Eq. (10) to a convex problem by changing the optimized variable and ignoring some constraints and solve it by the Lagrange dual method. Second, we re-consider the constraints ignored in the first step and formulate a linear programming problem to obtain the final solution for $P_{1}$ and $P_{2}$ based on the results in the first step.

\section{Step 1:}

Since Femtocell 1 and Femtocell 2 are adjacent, the interference from the adjacent femtocell is much more severe than the Gauss white noise power. Therefore, the Gauss white noise power can be ignored in the interference computation. Thus, Eqs. (7) and (8) are changed to Eqs. (16) and (17):

$$
\begin{aligned}
& \mathrm{Cap}_{1}= \\
& \int_{0}^{R_{1}} \int_{0}^{2 \pi} \frac{\rho_{1} P_{1} H_{1} C_{1} L_{1}^{-\alpha_{1}}}{P_{2} H_{2} C_{2}\left(\left(D-L_{1} \cos \theta\right)^{2}+\left(L_{1} \sin \theta\right)^{2}\right)^{-\frac{\alpha_{2}}{2}}} L_{1} d \theta d L_{1},
\end{aligned}
$$




$$
\begin{aligned}
& \mathrm{Cap}_{2}= \\
& \int_{0}^{R_{2}} \int_{0}^{2 \pi} \frac{\rho_{2} P_{2} H_{2} C_{2} L_{2}^{-\alpha_{2}}}{P_{1} H_{1} C_{1}\left(\left(D-L_{2} \cos \theta\right)^{2}+\left(L_{2} \sin \theta\right)^{2}\right)^{-\frac{\alpha_{1}}{2}}} L_{2} d \theta d L_{2} .
\end{aligned}
$$

For simplicity, we define $\mathrm{Co}_{1}$ and $\mathrm{Co}_{2}$ as Eqs. (18) and (19) to substitute the corresponding parts in Eqs. (16) and (17):

$$
\mathrm{Co}_{1}=\int_{0}^{R_{1}} \int_{0}^{2 \pi} \frac{\rho_{1} H_{1} C_{1} L_{1}^{-\alpha_{1}}}{H_{2} C_{2}\left(\left(D-L_{1} \cos \theta\right)^{2}+\left(L_{1} \sin \theta\right)^{2}\right)^{-\frac{\alpha_{2}}{2}}} L_{1} d \theta d L_{1}
$$

$\mathrm{Co}_{2}=\int_{0}^{R_{2}} \int_{0}^{2 \pi} \frac{\rho_{2} H_{2} C_{2} L_{2}^{-\alpha_{2}}}{H_{1} C_{1}\left(\left(D-L_{2} \cos \theta\right)^{2}+\left(L_{2} \sin \theta\right)^{2}\right)^{-\frac{\alpha_{1}}{2}}} L_{2} d \theta d L_{2}$

Then, Eqs. (16) and (17) are simplified to Eqs. (20) and (21):

$$
\begin{aligned}
& \operatorname{Cap}_{1}=\operatorname{Co}_{1} \frac{P_{1}}{P_{2}}, \\
& \mathrm{Cap}_{2}=\operatorname{Co}_{2} \frac{P_{2}}{P_{1}} .
\end{aligned}
$$

Then, Eqs. (10), (11), and (12) are changed to Eqs. (24) to (24):

$$
\max _{P_{1}, P_{2}}\left(\operatorname{Co}_{1} \frac{P_{1}}{P_{2}}+\operatorname{Co}_{2} \frac{P_{2}}{P_{1}}\right)
$$

subject to

$$
\begin{aligned}
& \mathrm{Co}_{1} \frac{P_{1}}{P_{2}} \geq \mathrm{Th}_{1} \\
& \mathrm{Co}_{2} \frac{P_{2}}{P_{1}} \geq \mathrm{Th}_{2}
\end{aligned}
$$

At this stage, we do not consider the constraints (13) to (15) temporarily. We substitute $\frac{P_{1}}{P_{2}}$ by the new optimized variable $\operatorname{Pr}$ in the optimization objective (22) and its constraints (23) and (24), and consequently, a new optimization expression is obtained as follows:

$$
\max _{\operatorname{Pr}}\left(\mathrm{Co}_{1} \operatorname{Pr}+\mathrm{Co}_{2} \frac{1}{\operatorname{Pr}}\right)
$$

subject to

$$
\begin{aligned}
& \mathrm{Co}_{1} \mathrm{Pr} \geq \mathrm{Th}_{1} \\
& \mathrm{Co}_{2} \frac{1}{\mathrm{Pr}} \geq \mathrm{Th}_{2}
\end{aligned}
$$

The problem (25) is a convex optimization problem with linear constraints. Therefore, we can adopt the Lagrange dual method to solve it. The solving process is shown in Appendix 1, where the optimal solution $\operatorname{Pr}_{o}$ for $\operatorname{Pr}$ variable is achieved.

\section{Step 2:}

At this stage, we will obtain the final optimal value for $P_{1}$ and $P_{2}$ based on the value of $\operatorname{Pr}_{o}$ achieved in step 1. From the point of energy saving, we aim to minimize the power consumption summation in the final solution of $P_{1}$ and $P_{2}$. Then, the problem required to be solved at this stage can be formulated as Eq. (28) with constraints (29) to (34).

$$
\min _{P_{1}, P_{2}}\left(P_{1}+P_{2}\right)
$$

subject to

$$
\frac{P_{1}}{P_{2}}=\operatorname{Pr}_{o}
$$

$\int_{0}^{R_{1}} \int_{0}^{2 \pi} \frac{\rho_{1} P_{1} H_{1} C_{1} L_{1}^{-\alpha_{1}}}{P_{2} H_{2} C_{2}\left(\left(D-L_{1} \cos \theta\right)^{2}+\left(L_{1} \sin \theta\right)^{2}\right)^{-\frac{\alpha_{2}}{2}}+\sigma^{2}} L_{1} d \theta d L_{1} \geq \mathrm{Th}_{1}$

$\int_{0}^{R_{2}} \int_{0}^{2 \pi} \frac{\rho_{2} P_{2} H_{2} C_{2} L_{2}^{-\alpha_{1}}}{P_{1} H_{1} C_{1}\left(\left(D-L_{2} \cos \theta\right)^{2}+\left(L_{2} \sin \theta\right)^{2}\right)^{-\frac{\alpha_{2}}{2}}+\sigma^{2}} L_{2} d \theta d L_{2} \geq \mathrm{Th}_{2}$

$$
\begin{aligned}
& P_{1} \leq P_{\mathrm{LT} 1} \\
& P_{2} \leq P_{\mathrm{LT} 2} \\
& P_{1} h_{1 p}+P_{2} h_{2 p} \leq I_{p}
\end{aligned}
$$

Constraints (30) and (31) mean that with both consideration of the adjacent femtocell interference and Gauss white noise, the capacity of Femtocell 1 and Femtocell 2 needs to exceed to the respective minimal capacity requirements. Constraints (32) to (34) limit the maximal permitted value for $P_{1}$ and $P_{2}$. But we cannot obtain the lower limitation of $P_{1}$ and $P_{2}$ directly from Eqs. (32) to (34). However, the transmit power of the base station must be large enough to meet the capacity requirements of the cell. Hence, we analyze the variation relation between $P_{1}$ and $P_{2}$ according to Eqs. (30) and (31) so as to obtain lower limits of $P_{1}$ and $P_{2}$, which leads to Theorem 1 .

Theorem 1. By adhering to the solution of problem (25), i.e., the ratio of $P_{1}$ and $P_{2}$ keeps at a specific value of $\mathrm{Pr}_{o}$, we can conclude that the following two constraints are equivalent to constraints (30) and (31), respectively.

$$
\begin{aligned}
& P_{2} \geq \frac{M_{0} \sigma^{2}}{H_{2} C_{2}\left(D+R_{1}\right)^{-\alpha_{2}}}, \\
& P_{1} \geq \frac{M_{0} \sigma^{2}}{H_{1} C_{1}\left(D+R_{2}\right)^{-\alpha_{1}}},
\end{aligned}
$$

where $M_{0}$ is a relatively large value which makes the noise part in the denominator of Eqs. (30) and (31) ignorable. 
Proof. Before going to the detailed proof, we would like to introduce a common skill in mathematics. Define two real variables $X$ and $Y$ and a relatively large value of $M$. If $X \geq M Y$ is satisfied, we say that $Y$ is small enough to be ignored in the expression of $X+Y$. Then, it is sufficient to prove that we can find a value of $M_{0}$ which makes the noise part in the denominator of Eqs. (30) and (31) ignorable.

Let us take the deduction of the lower limit of $P_{2}$ as example and analyze the relative variation process of $P_{1}$ and $P_{2}$ in detail. Firstly, we assume that the transmission power $P_{2}$ is equal to zero. Then, constraint (30) is changed to Eq. (37), which indicates that the transmission power of Femtocell 1 only needs to resist the interference of Gauss white noise power to meet the capacity requirement of $T h_{1}$,i.e.,

$$
\int_{0}^{R_{1}} \int_{0}^{2 \pi} \frac{\rho_{1} P_{1} H_{1} C_{1} L_{1}^{-\alpha_{1}}}{\sigma^{2}} L_{1} d \theta d L_{1} \geq \mathrm{Th}_{1}
$$

We define $P_{\mathrm{LT} 1}^{\prime}$ to indicate the minimal $P_{1}$ value, which makes the capacity requirement $\mathrm{Th}_{1}$ satisfied when the transmission power of Femtocell 2 is zero. Then, Eq. (38) is obtained, and it can be concluded that $P_{1} \geq P_{\mathrm{LT} 1}^{\prime}$ is always satisfied.

$$
\int_{0}^{R_{1}} \int_{0}^{2 \pi} \frac{\rho_{1} P_{\mathrm{LT} 1}^{\prime} H_{1} C_{1} L_{1}^{-\alpha_{1}}}{\sigma^{2}} L_{1} d \theta d L_{1}=\mathrm{Th}_{1}
$$

Secondly, we consider the scenario that $P_{2}$ increases from zero. When $P_{2}$ starts to be larger than zero, $P_{1}$ needs to be larger than $P_{\mathrm{LT} 1}^{\prime}$ and changes to $\operatorname{Pr}_{o} P_{2}$ in order to satisfy constraint (29). Then, since Eq. (29) is the solution of problem (22) according to step 1, it can be concluded that Eq. (29) can make Eq. (23) satisfied, which means that $\mathrm{Th}_{1}$ is attained. But in problem (22), Gauss white noise power is ignored, whereas in Eq. (30) the interference of Gauss white noise power is considered; therefore, $P_{1}$ must be larger than $\operatorname{Pr}_{o} P_{2}$ to make Eq. (30) satisfied. Then, we define $P_{1}^{\prime}$ indicating the new value of $P_{1}$ which is larger than $\operatorname{Pr}_{o} P_{2}$ to make Eq. (30) satisfied. Consequently, as Eq. (31) must be satisfied for the solution of Eq. (28), $P_{2}$ will increase with $P_{1}^{\prime}$ to $P_{2}^{\prime}$, which make $P_{1}^{\prime}=\operatorname{Pr}_{o} P_{2}^{\prime}$ exist. Repeatedly, $P_{1}$ will increase again because of the consideration of Gauss white noise power and $P_{2}$ will increase accordingly by the constraint (29). The process will be repeated until $P_{2}$ is large enough to make $\sigma^{2}$ insignificant. Then, constraint (30) becomes in accordance with Eq. (23), and Eq. (29) can make Eq. (30) satisfied.

From Eq. (2) and Fig. 2, it can be seen that when $L_{2}$ equals to $D+R_{1}$, the $P_{2}$ interference to Femtocell 1 arrives at the smallest value of the Femtocell 1 coverage area. If the smallest $P_{2}$ interference to Femtocell 1 is large enough to make $\sigma^{2}$ insignificant, we can ignore $\sigma^{2}$ in all the coverage areas of Femtocell 1. Well then, according to Eqs. (3) and (4), it can be concluded that when Eq. (39) is satisfied we can ignore the interference of $\sigma^{2}$ for all coverage of Femtocell 1.

$$
P_{2} H_{2} C_{2}\left(D+R_{1}\right)^{-\alpha_{2}} \geq M_{0} \sigma^{2}
$$

Since, when $\sigma^{2}$ is ignored, constraint (29) can make Eq. (30) satisfied and constraint (30) can be transformed to Eq. (39) equivalently. From Eq. (39), Eq. (35) is proved, which gives the lower limit of $P_{2}$. The process described above is shown in Fig. 3.

A similar process can be applied to obtain the $P_{1}$ lower limit, and Eq. (36) can be proved.

By Theorem 1 usage, the optimization problem (28) is transformed into a convex linear programming problem. The solution process is shown in Appendix 2, in which we obtain the final solution for $P_{1}$ and $P_{2}$.

\subsection{Detailed procedure of algorithm}

The whole process of obtaining the final value of $P_{1}$ and $P_{2}$ is illustrated in Algorithm 1. Firstly, we obtain the $\operatorname{Pr}_{o}$ value of the problem (25) solution according to step 1 in Section 3.3. Then, for step 2, according to the proof of Theorem 1, we perform two iterative processes to obtain

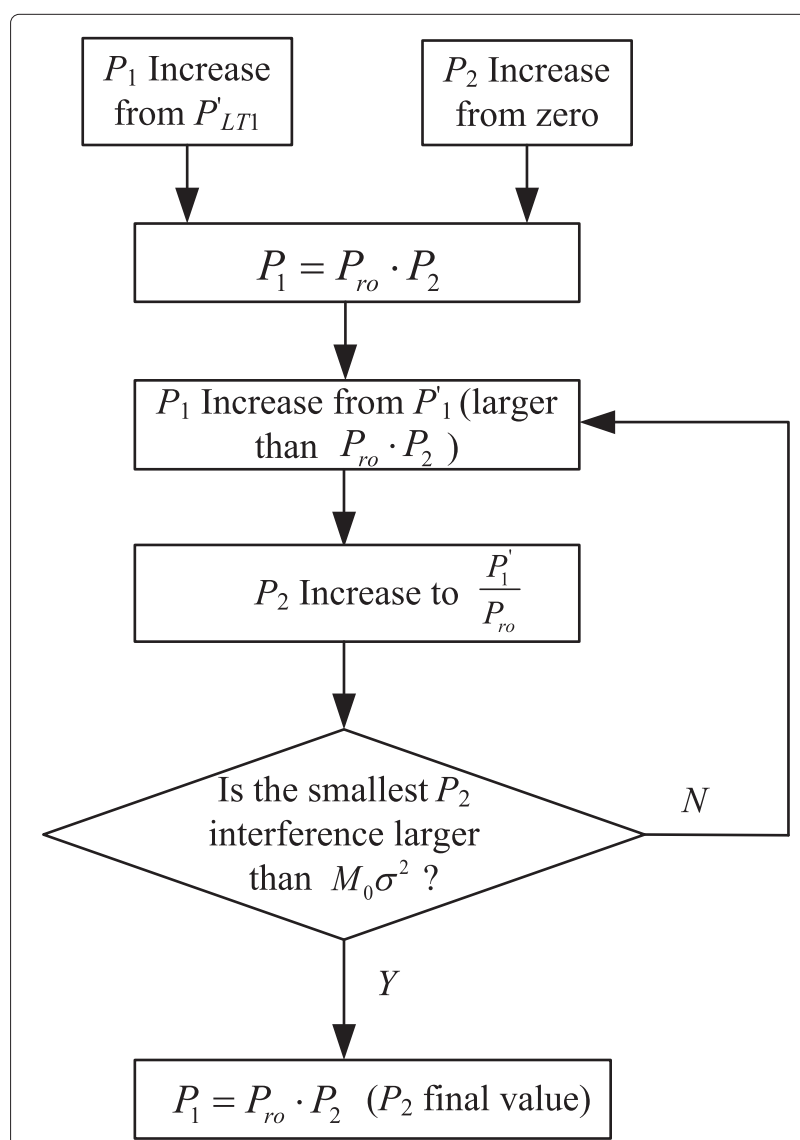

Fig. 3 Variation process of $P_{1}$ and $P_{2}$ 


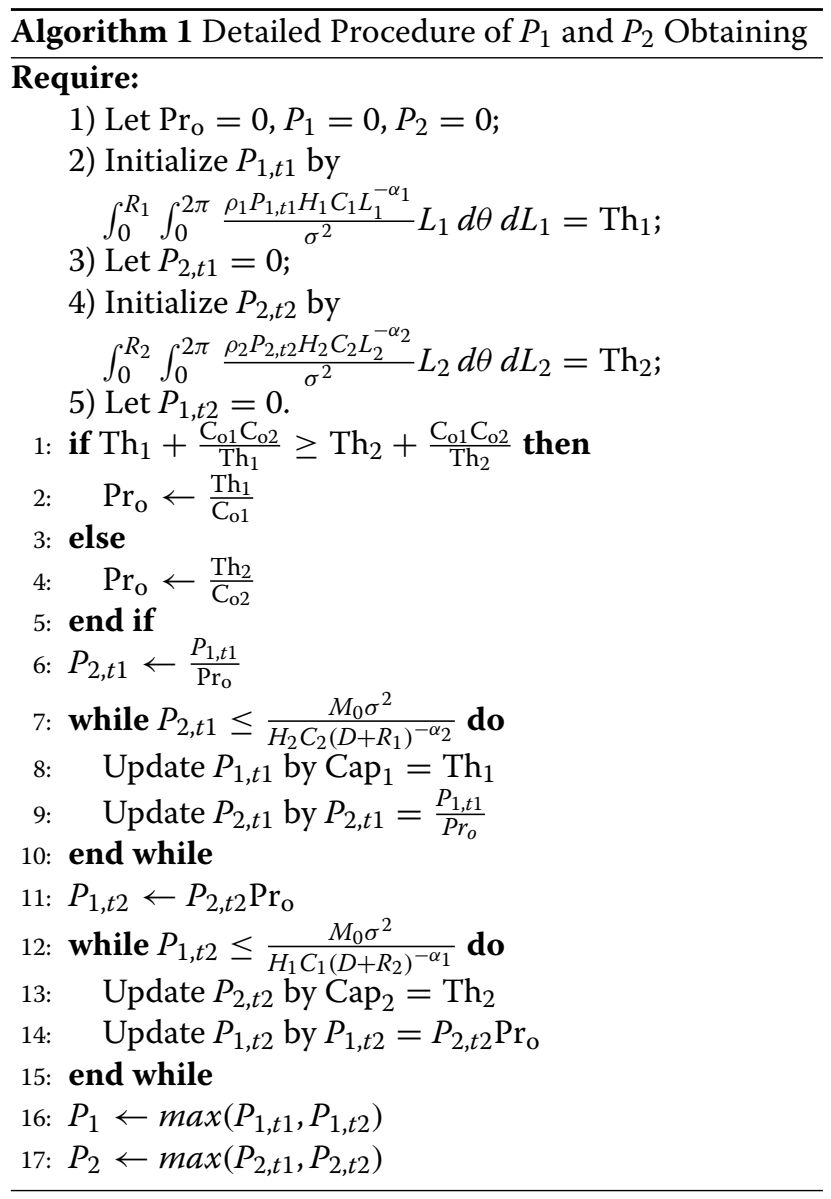

two groups of $P_{1}$ and $P_{2}$ values, which satisfy the lowest constraints of $P_{1}$ and $P_{2}$, respectively. The two group values are denoted by $P_{1, t 1}$ and $P_{2, t 1}$ and $P_{1, t 2}$ and $P_{2, t 2}$, respectively. Lastly, since the lowest constraints of $P_{1}$ and $P_{2}$ need to be satisfied simultaneously, we choose the maximal values of the two groups as the final result for $P_{1}$ and $P_{2}$.

\section{Simulation results}

In this section, we validate the performance of the proposed power management scheme for adjacent femtocells by Monte Carlo simulations. We build a two-layer heterogeneous network with one macro cell and two adjacent femtocells as shown in Figs. 1 and 2. We consider twoband usage $(800 \mathrm{MHz}$ and $2.5 \mathrm{GHz})$ as in [15]. The simulation parameters and their numerical values are summarized in Table 1.

\subsection{Analysis of influence of $P_{1}$ and $P_{2}$ variation upon capacity}

We validate the influence of $P_{1}$ and $P_{2}$ variation upon the sum capacity computed by (9). We choose two groups of setting for $\left[\rho_{1}, \rho_{2}\right]$ for comparison, which are $[100,100]$ and $[100,200]$. The value of $\left[R_{1}, R_{2}\right]$ is set to $[0.6,0.6]$ for
Table 1 Numerical values of simulation parameters

\begin{tabular}{|c|c|}
\hline Parameters & Values \\
\hline Gauss white noise power $\sigma^{2}$ & $-80 \mathrm{dbW}$ \\
\hline Fading value $H_{1}$ & Rayleigh fading ( $\sim \operatorname{Exp}(1))$ \\
\hline Fading value $\mathrm{H}_{2}$ & Rayleigh fading ( Exp (1)) \\
\hline $800 \mathrm{MHz}$ path loss constant $C_{1}$ & $\left(\frac{0.375}{4 \pi}\right)^{2}$ \\
\hline $800 \mathrm{MHz}$ path loss constant $C_{2}$ & $\left(\frac{0.375}{4 \pi}\right)^{2}$ \\
\hline $800 \mathrm{MHz}$ path loss exp. $\alpha_{1}$ & 3 \\
\hline $800 \mathrm{MHz}$ path loss exp. $\alpha_{2}$ & 3 \\
\hline $2.5 \mathrm{GHz}$ path loss constant $C_{1}$ & $\left(\frac{0.12}{4 \pi}\right)^{2}$ \\
\hline $2.5 \mathrm{GHz}$ path loss constant $C_{2}$ & $\left(\frac{0.12}{4 \pi}\right)^{2}$ \\
\hline $2.5 \mathrm{GHz}$ path loss exp. $\alpha_{1}$ & 4 \\
\hline $2.5 \mathrm{GHz}$ path loss exp. $\alpha_{2}$ & 4 \\
\hline Variable comparison parameter $M_{0}$ & 10 \\
\hline Femtocell 1 capacity requirements $T_{1}$ & $200 \mathrm{Mbit} / \mathrm{s}$ \\
\hline Femtocell 2 capacity requirements $\mathrm{Th}_{2}$ & $500 \mathrm{Mbit} / \mathrm{s}$ \\
\hline Femtocell 1 user distribution density $\rho_{1}$ & $100 / \mathrm{km}^{2}$ \\
\hline Femtocell 2 user distribution density $\rho_{2}$ & $100 / \mathrm{km}^{2}, 200 / \mathrm{km}^{2}$ \\
\hline Femtocell 1 maximal power $P_{\mathrm{LT} 1}$ & $1 \mathrm{~W}$ \\
\hline Femtocell 2 maximal power $P_{\mathrm{LT} 2}$ & $2 W$ \\
\hline Femtocell 1 interference gain $h_{1 p}$ & Rayleigh fading ( $\sim \operatorname{Exp}(1))$ \\
\hline Femtocell 2 interference gain $h_{2 p}$ & Rayleigh fading ( $\sim \operatorname{Exp}(1))$ \\
\hline Licensed network interference constraint $I_{p}$ & $10 \mathrm{~W}$ \\
\hline Femtocell 1 and Femtocell 2 distance $D$ & $1 \mathrm{~km}$ \\
\hline
\end{tabular}

both groups of setting. Figures 4 and 5 shows the capacity changes with $P_{1}$ and $P_{2}$ variation corresponding to the two groups of setting. From the two figures it can be seen that in the middle part the capacity value is lower and in the edge part the capacity value is higher. In other words the variation trend can be summarized as the sum capacity increases with the difference value of $P_{1}$ and $P_{2}$ enlarging.

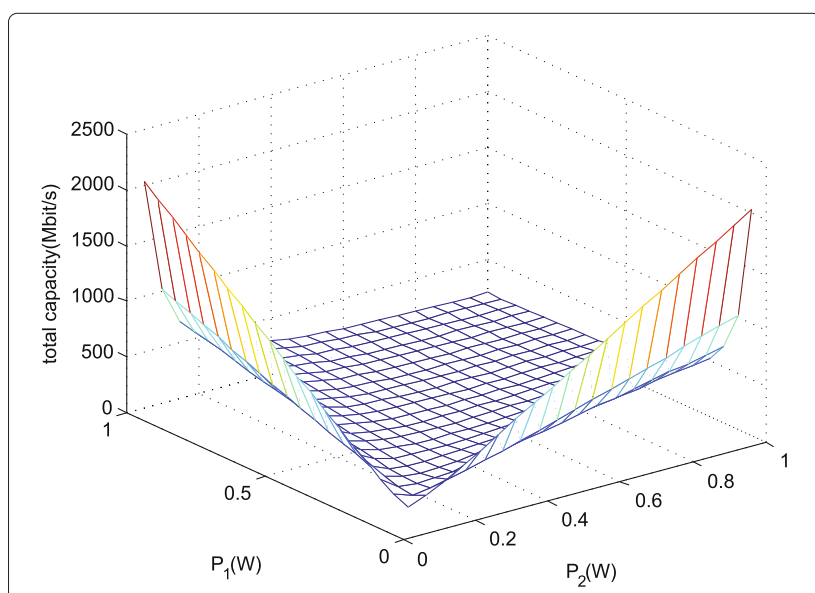

Fig. 4 Capacity changing with $P_{1}$ and $P_{2}\left(\left[\rho_{1}, \rho_{2}\right]\right.$ set to $\left.[100,100]\right)$ 


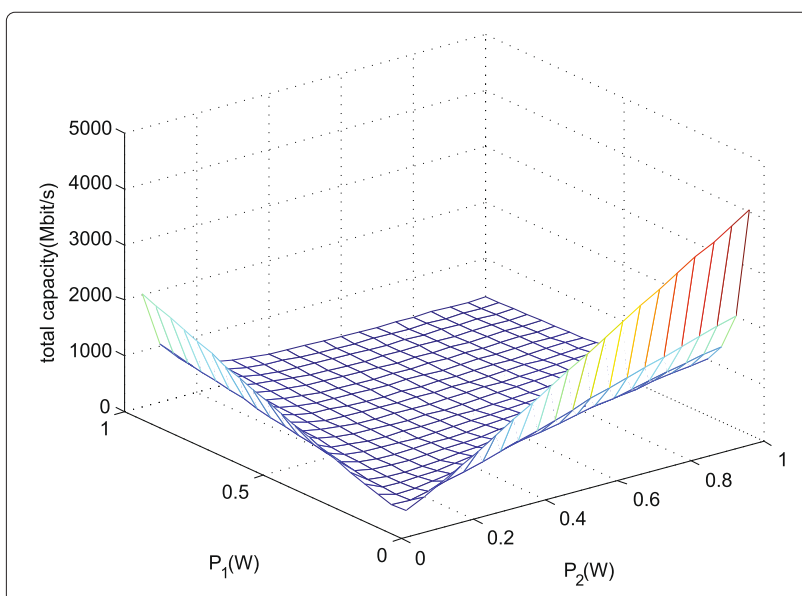

Fig. 5 Capacity changing with $P_{1}$ and $P_{2}\left(\left[\rho_{1}, \rho_{2}\right]\right.$ set to $\left.[100,200]\right)$

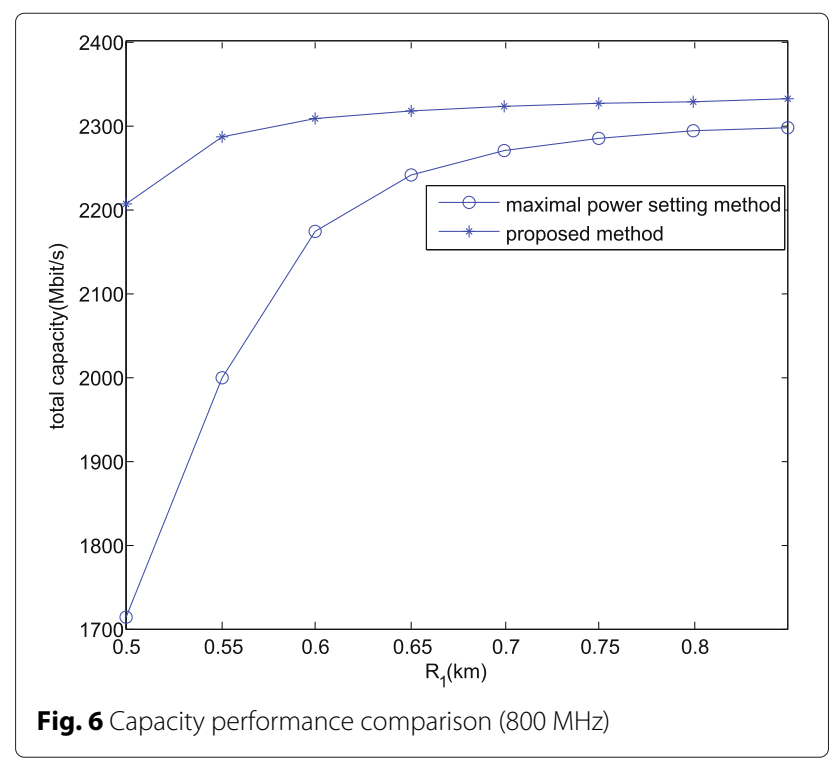

\subsection{Energy performance analysis of proposed method}

The energy consumption has been one of the main concerns in the current researches of wireless communication system. In the proposed power management method, besides the network capacity maximization, we look into energy consumption minimization in problem (26). Therefore, we validate the power consumption performance of the proposed method by comparing it with the maximal power setting method as shown in Fig. 8. For Fig. 8 , we set $R_{2}$ as $0.5 \mathrm{~km}$ and $\left[\rho_{1}, \rho_{2}\right]$ as $[100,100]$. In Fig. 8 , we take into account $800-\mathrm{MHz}$ and $2-\mathrm{GHz}$ usage. It is shown that in the proposed method the power consumption increases with the $R_{1}$ value. This is because, for power consumption minimization, $P_{1}$ and $P_{2}$ will be set to the lower limit as far as possible, and we obtain the lower limit by Eqs. (35) and (36), which increase as $R_{1}$ and

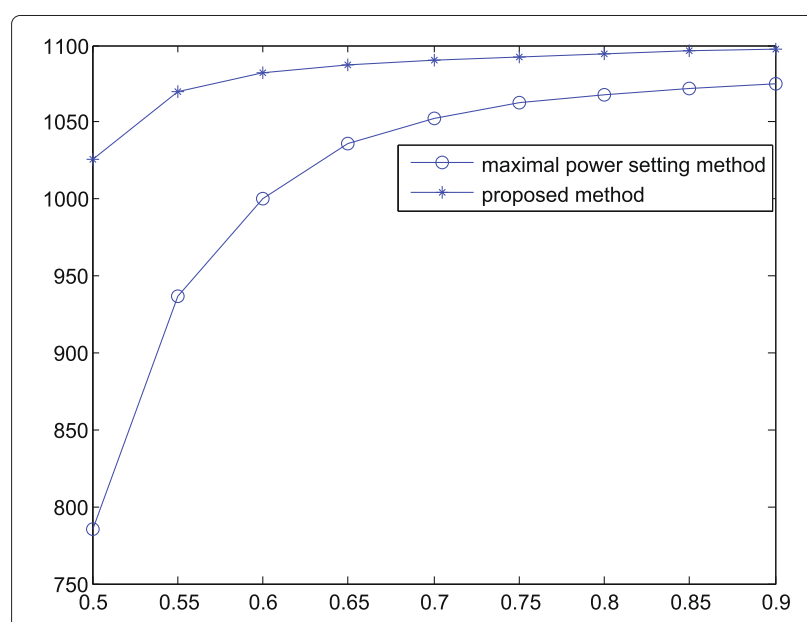

Fig. 7 Capacity performance comparison $(2.5 \mathrm{GHz})$ power to the maximal limitation. 


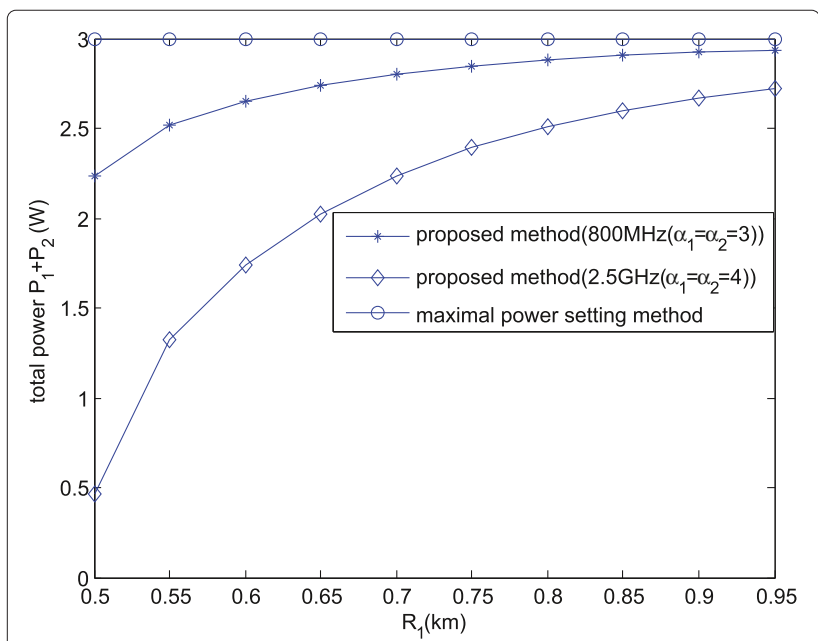

Fig. 8 Energy consumption performance comparison

$R_{2}$ increase. Therefore, when $R_{2}$ is fixed, the sum power increases with $R_{1}$ variation until it becomes infinitely close to the upper limit.

\section{Conclusions}

We propose a two-stage power configuration scheme for adjacent femtocells in cognitive heterogeneous network in this paper. In the first stage, by jointly considering the distance-dependent interference, user distribution, and user performance in terms of SNR, we manage to derive an expression for sum capacity of femtocells. Subsequently, we formulate the problem of maximizing sum capacity while satisfying the minimum capacity requirements for each femtocell. The solution for this problem indicates that maximum sum capacity can be achieved as long as the ratio between the transmission power of two adjacent femtocells keeps at some specific value. Then, in the second stage, we further formulate a new problem of minimizing power consumption while adhering to the solution in the first stage. Optimal power setting for each femtocell is derived in this stage. Extensive simulations show that the proposed scheme brings significant enhancement in terms of network capacity and energy conservation. In the future, we plan to extend our research to the scenario of multiple adjacent femtocells.

\section{Appendix 1}

Proof. For problem (25), we define the Lagrange dual function as Eq. (40):

$$
\begin{aligned}
& L(x, \lambda)= \\
& -\left(\mathrm{Co}_{1} \operatorname{Pr}+\mathrm{Co}_{2} \frac{1}{\mathrm{Pr}}\right)+\lambda_{1}\left(\mathrm{Th}_{1}-\mathrm{Co}_{1} \mathrm{Pr}\right) \\
& +\lambda_{2}\left(\mathrm{Th}_{2}-\mathrm{Co}_{2} \frac{1}{\mathrm{Pr}}\right)
\end{aligned}
$$

in which, $\lambda_{i}(i=1,2)$ is the Lagrange multiplier corresponding to constraints (26) and (27). Then, the KKT (Karush-Kuhn-Tucker) conditions of the problem (25) can be expressed as following from Eqs. (41) to (46):

$$
\begin{aligned}
& \mathrm{Co}_{1} \operatorname{Pr} \geq \mathrm{Th}_{1} \\
& \mathrm{Co}_{2} \frac{1}{\operatorname{Pr}} \geq \mathrm{Th}_{2} \\
& \lambda_{i} \geq 0(i=1,2) \\
& \lambda_{1}\left(\mathrm{Th}_{1}-\mathrm{Co}_{1} \operatorname{Pr}\right)=0 \\
& \lambda_{2}\left(\mathrm{Th}_{2}-\mathrm{Co}_{2} \frac{1}{\mathrm{Pr}^{2}}\right)=0 \\
& -\left(\mathrm{Co}_{1}+\mathrm{Co}_{2} \frac{1}{\mathrm{Pr}^{2}}\right)-\lambda_{1} \mathrm{Co}_{1}+\lambda_{2} \mathrm{Co}_{2} \frac{1}{\mathrm{Pr}^{2}}=0
\end{aligned}
$$

From KKT conditions, we can obtain the possible solution as Eq. (47):

$$
\left\{\begin{array}{l}
\operatorname{Pr}_{1}=\frac{\mathrm{Th}_{1}}{\mathrm{Co}_{1}} \\
\operatorname{Pr}_{2}=\frac{\mathrm{Co}_{2}}{\mathrm{Th}_{2}}
\end{array}\right.
$$

Bring $\operatorname{Pr}_{1}$ and $\operatorname{Pr}_{2}$ to Eq. (25), then we can obtain the final solution of Pr as Eq. (48):

$$
\operatorname{Pr}=\left\{\begin{array}{l}
\frac{\mathrm{Th}_{1}}{\mathrm{Co}_{1}} \mathrm{Th}_{1}+\frac{\mathrm{Co}_{1} \mathrm{Co}_{2}}{\mathrm{Th}_{1}} \geq \mathrm{Th}_{2}+\frac{\mathrm{Co}_{1} \mathrm{Co}_{2}}{\mathrm{Th}_{2}} \\
\frac{\mathrm{Co}_{2}}{\mathrm{Th}_{2}} \mathrm{Th}_{1}+\frac{\mathrm{Co}_{1} \mathrm{Co}_{2}}{\mathrm{Th}_{1}} \leq \mathrm{Th}_{2}+\frac{\mathrm{Co}_{1} \mathrm{Co}_{2}}{\mathrm{Th}_{2}}
\end{array}\right.
$$

\section{Appendix 2}

Proof. We substitute Eqs. (30) and (31) with Eqs. (35) and (36), respectively, and problem (28) becomes a linear programming program. Afterwards, we eliminate one variable, $P_{1}$, and the problem (28) is transformed to Eq. (49):

$$
\min _{P_{2}}\left(\left(1+\operatorname{Pr}_{o}\right) P_{2}\right)
$$

subject to

$$
\begin{aligned}
P_{2} & \geq \frac{M_{0} \sigma^{2}}{H_{2} C_{2}\left(D+R_{1}\right)^{-\alpha_{2}}} \\
P_{2} & \geq \frac{M_{0} \sigma^{2}}{H_{1} C_{1}\left(D+R_{2}\right)^{-\alpha_{1}} \operatorname{Pr}_{o}} \\
P_{2} & \leq \frac{P_{\mathrm{LT} 1}}{\operatorname{Pr}_{o}} \\
P_{2} & \leq P_{\mathrm{LT} 2} \\
P_{2} & \leq \frac{I_{p}}{\operatorname{Pr}_{o} h_{1 p}+h_{2 p}}
\end{aligned}
$$


Equations (50) to (51) give the permitted range of $P_{2}$, and Eqs. (52) to (54) indicate the lower limit of $P_{2}$. The optimal solution for $P_{2}$ can be obtained as Eq. (55):

$$
P_{2}=\max \left(\frac{M_{0} \sigma^{2}}{H_{2} C_{2}\left(D+R_{1}\right)^{-\alpha_{2}}}, \frac{M_{0} \sigma^{2}}{H_{1} C_{1}\left(D+R_{2}\right)^{-\alpha_{1}} \operatorname{Pr}_{o}}\right)
$$

The corresponding solution for $P_{1}$ is obtained as Eq. (56):

$$
P_{1}=\operatorname{Pr}_{o} \max \left(\frac{M_{0} \sigma^{2}}{H_{2} C_{2}\left(D+R_{1}\right)^{-\alpha_{2}}}, \frac{M_{0} \sigma^{2}}{H_{1} C_{1}\left(D+R_{2}\right)^{-\alpha_{1}} \operatorname{Pr}_{o}}\right)
$$

\section{Competing interests}

The authors declare that they have no competing interests.

\section{Acknowledgements}

This work is supported by the National Natural Science Foundation of China (NSFC) (Grant No. 61402107), Doctoral Scientific Research Foundation of Guangdong Province (Grant No. ZJ121204), Natural Science Foundation of Guangdong Province (Grant No. 2015A030313652) and Guangdong Province Commonweal Research Technological Project (Grant No. 2015A010103019, 2015A010103020)

Received: 24 August 2015 Accepted: 20 December 2015

Published online: 08 January 2016

\section{References}

1. H Zhang, C Jiang, N Beaulieu, X Chu, X Wang, T Quek, Resource allocation for cognitive small cell networks: a cooperative bargaining game theoretic approach. IEEE Trans. Wirel. Commun. 14, 3481-3493 (2015)

2. JMR Avil, M Toril, S L-Ramirez, A femtocell location strategy for improving adaptive traffic sharing in heterogeneous LTE networks. EURASIP J. Wirel. Commun. Netw. 12, 1-13 (2015)

3. H Zhang, C Jiang, X Mao, H Chen, Interference-limited resource optimization in cognitive femtocells with fairness and imperfect spectrum sensing. IEEE Trans. Veh. Technol. 10, 1-11 (2015)

4. F Song, X Wen, N Li, Power allocation for OFDM-based cognitive heterogeneous networks. SCIENCE CHINA. 58, 1-12 (2013)

5. F Chen, W Xu, Y Guo, J Lin, M Chen, in Proceedings of the 8th International Conference on Communications and Networking in China: August 2013; Guilin. Resource allocation in OFDM-based heterogeneous cognitive radio networks with imperfect spectrum (IEEE, 2013), pp. 46-51

6. SCheng, S Lien, F Chu, K Chen, On exploiting cognitive radio to mitigate interference in macro/femto heterogeneous networks. IEEE Wirel. Commun. 18, 40-47 (2011)

7. G Jagadish, R Catherine, Resource allocation, transmission coordination and user association in heterogeneous networks a flow-based unified approach. IEEE Trans. Wirel. Commun. 12, 1340-1351 (2013)

8. M Adian, H Aghaeinia, Optimal resource allocation in heterogeneous MIMO cognitive radio networks. IEEE Wireless Personal Commun. 76, 23-39 (2014)

9. S Wang, Z Zhou, M Ge, C Wang, Power allocation for OFDM-based cognitive heterogeneous networks. IEEE J. Sel. Areas Commun. 31, 464-475 (2013)

10. R Xie, F Yu, H Ji, Y Li, Energy-efficient resource allocation for heterogeneous cognitive radio networks with femtocells. IEEE Trans. Wirel. Commun. 11, 3910-3920 (2011)

11. S Bu, F Yu, Y Qian, in Proceedings of the 31st International Conference on Computer Communications: December 2012; Anaheim. Energy-efficient cognitive heterogeneous networks powered by the smart grid (IEEE, New York, NY, USA, 2012), pp. 23-39
12. X Peng, G Peng, J Hyun-Park, D Park, D Ky-Kim, in Proceedings of the 9th International Symposium on Communications and Information Technology: September 2009; Icheon. Max-min fairness based radio resource management in fourth generation heterogeneous networks (IEEE, New York, NY, USA, 2009), pp. 208-213

13. H Zhang, C Jiang, N Beaulieu, X Chu, X Wen, M Tao, Resource allocation in spectrum-sharing OFDMA femtocells with heterogeneous services. IEEE Trans. on Commun. 7, 2366-2377 (2014)

14. H Zhang, X Chu, W Guo, S Wang, Coexistence of wi-fi and heterogeneous small cell networks sharing unlicensed spectrum. IEEE Commun. Mag. 53, 158-164 (2015)

15. H Zhang, H Liu, C Jiang, X Chu, A Nallanathan, X Wen, A practical semi-dynamic clustering scheme using affinity propagation in cooperative picocells. IEEE Trans. Veh. Technol. 99, 1-11 (2014)

16. X Lin, J Andrews, A Ghosh, Analysis and design for carrier aggregation in heterogeneous cellular networks. IEEE Trans. Commun. 61, 4002-4015 (2013)

\section{Submit your manuscript to a SpringerOpen ${ }^{\circ}$ journal and benefit from:}

- Convenient online submission

- Rigorous peer review

- Immediate publication on acceptance

- Open access: articles freely available online

- High visibility within the field

- Retaining the copyright to your article

Submit your next manuscript at $>$ springeropen.com 